\title{
A taxonomic review of the Gyrinidae (Coleoptera) in Korea
}

\author{
Dae-Hyun Lee', Kee-Jeong Ahn' \\ I Department of Biology, Chungnam National University, Daejeon 305-764, Republic of Korea \\ Corresponding author: Kee-Jeong Ahn (kjahn@cnu.ac.kr)
}

Academic editor: M. Fikácek | Received 24 February 2015 | Accepted 6 June 2015 | Published 24 June 2015

http://zoobank.org/3F6A7DB7-36A8-47E8-846A-A7B2787039D5

Citation: Lee D-H, Ahn K-J (2015) A taxonomic review of the Gyrinidae (Coleoptera) in Korea. ZooKeys 509: 87-107. doi: $10.3897 /$ zookeys.509.9442

\begin{abstract}
A taxonomic review of Korean Gyrinidae is presented. Seven species [Dineutus orientalis (Modeer, 1776), Gyrinus gestroi Régimbart, 1883, G. japonicus Sharp, 1873, G. pullatus Zaitzev, 1908, Orectochilus punctipennis Sharp, 1884, O. Regimbarti Sharp, 1884 and O. villosus (Müller, 1776)] in three genera are recognized, one of which (O. punctipennis Sharp, 1884) is reported for the first time in Korea. We also found that $G$. curtus Motschulsky, 1866 previously recorded in Korea was an incorrect identification of $G$. pullatus Zaitzev, 1908. Habitus and SEM photographs, distribution maps, keys, and diagnoses of genera and species are provided.
\end{abstract}

\section{Keywords}

Taxonomy, Gyrinidae, Coleoptera, Korea

\section{Introduction}

The Gyrinidae are water beetles with unique swimming behavior where adults rapidly gyrate and whirl on the surface of water. They inhabit stagnant or slowly running water and prefer clean oxygen-rich habitats (Brinck 1955). Members of the Gyrinidae 
are characterized by the combination of the following characters: compound eyes divided completely with one pair on the dorsal surface of the head (above the water line) and another on the ventral surface of the head (below the water line); antenna short with a broad, cup-shaped scape, subtriangular pedicel, elongate but compact flagellum; middle and hind legs broadly expanded and fringed with setae for swimming (Miller and Bergsten 2012).

The whirligig beetles contain about 1000 species in 25 genera worldwide (Slipinski et al. 2011) and 118 species in seven genera in the Palaearctic region (Mazzoldi 2003). In Korea, seven species in three genera have been recorded (Jung et al. 2011), 50 species in six genera from China, 16 species in three genera from Japan and 11 species in three genera from the Far East of Russia (Mazzoldi 2003).

It was Kolbe (1886) who recorded the first gyrinid species, Gyrinus japonicus Sharp, 1873 in Korea. About 50 years later, Japanese entomologists, Takizawa (1931) and Kamiya (1936) reported two species (Dineutus orientalis Modeer, 1776 and G. japonicus Sharp, 1873) with descriptions and illustrations. Cho (1957) was the first Korean beetle taxonomist who studied Korean gyrinid fauna in detail. Since then, a few entomologists have studied Korean gyrinid beetles, mainly in the local fauna and no taxonomic review has been performed since Yoon (1988). Accordingly, this review is an updated contribution worth undertaking for Korean Gyrinidae.

In this paper we recognized seven species [D. orientalis (Modeer, 1776), G. gestroi Régimbart, 1883, G. japonicus Sharp, 1873, G. pullatus Zaitzev, 1908, Orectochilus punctipennis Sharp, 1884, O. Regimbarti Sharp, 1884 and O. villosus (Müller, 1776)]: O. punctipennis Sharp is identified for the first time in Korea and G. curtus Motschulsky previously recorded in Korea was an incorrect identification of $G$. pullatus Zaitzev. We provided habitus and SEM photographs, distribution maps, keys, and diagnoses of genera and species.

\section{Materials and methods}

To identify Korean Gyrinidae more reliably, we compared them with type and voucher specimens in the National History Museum (NHM, London, United Kingdom). The specimens used in this study are deposited in Chungnam National University Insect Collection (CNUIC), Daejeon, Korea and Ehime University Museum (EUMJ), Matsuyama, Japan. Habitus photographs were prepared from single or multi-layered shots taken with Olympus DP71 camera with several images amalgamated using Helicon Focus 5.3 (Helicon Soft Ltd, Kharkov, Ukraine) and edited by Adobe Photoshop CS4 (Adobe Systems, San Jose, CA, USA). Dry specimens for scanning electron microscope (SEM) photographs were sputter coated with platinum (Cressington 208 auto sputter coater, Hertfordshire, UK) and examined under SEM (S-4800, Hitachi, Tokyo, Japan). The terminology of taxonomic characters and measurements of specimens mainly follow Holmen (1987) and Miller and Bergsten (2012). The subdivision of China and Russia follows the standards of Löbl and Smetana (2003). 


\section{Results}

\section{Gyrinidae Latreille, 1810}

\section{Key to the genera of Korean Gyrinidae}

1 Pronotum with transverse depression on median part; elytra with rows of

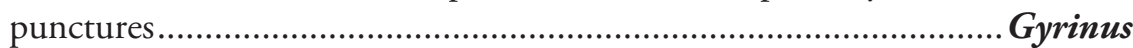

- $\quad$ Pronotum without transverse depression; elytra without rows of punctures... 2

2 Labrum short and broad; elytra without compact setae.................. Dineutus

- $\quad$ Labrum long and subtriangular; elytra with compact setae........ Orectochilus

\section{Genus Dineutus MacLeay, 1825}

Figs $7-8,10-14$

Dineutus MacLeay, 1825: 133. Type species: Dineutus politus MacLeay, 1825.

Diagnosis. Head about 2.0 times as wide as long, with polygonal micro-reticulation. Clypealium with long setae. Antenna with 6 antennomeres. Galea absent; cardo and stipes with few setae on apico-lateral part. Pronotum (Fig. 7) convex without transverse groove; anterior margin bisinuate, posterior margin rounded, anterior angle acute, posterior angle nearly rectangular. Scutellum (Fig. 8) concealed when elytra closed. Elytra (Fig. 8) widest at middle, without punctato-striae and compact setae; subelytral suture absent. Prosternum (Fig. 10) transverse; anterior margin rounded; prosternal process (Fig. 10) linear-form and posterior margin rounded. Anterior margin of mesoventrite (Fig. 11) acute, posterior part bifid. Metaventrite (Fig. 12) broad, diamond-shaped, flattened; anterior part acute, lateral parts transverse. Sternite II (Fig. 13) without pit and groove. Sternite VIII (Fig. 14) with compact and short setae on lateral margins.

\section{Subgenus Spinosodineutes Hatch, 1925: 447}

Spinosodineutes Hatch, 1925: 447. Type species: Gyrinus spinosus Fabricius, 1781. Gyrinodineutus Ochs, 1926: 66. Type species: Dineutus unidentatus Aubé, 1838. Synonymized by Brinck (1955: 104).

\section{Dineutus (Spinosodineutes) orientalis (Modeer, 1776)}

Figs 1, 7-14, 41-43, 59

Gyrinus orientalis Modeer, 1776: 160.

Dineutus marginatus Sharp, 1873: 56. Synonymized by Ochs (1926: 136).

Dineutus quadrispina Fairmaire, 1878: 88. Synonymized by Ahlwarth (1910: 6).

Dineutus (Spinosodineutes) orientalis: Ochs (1930: 9). 

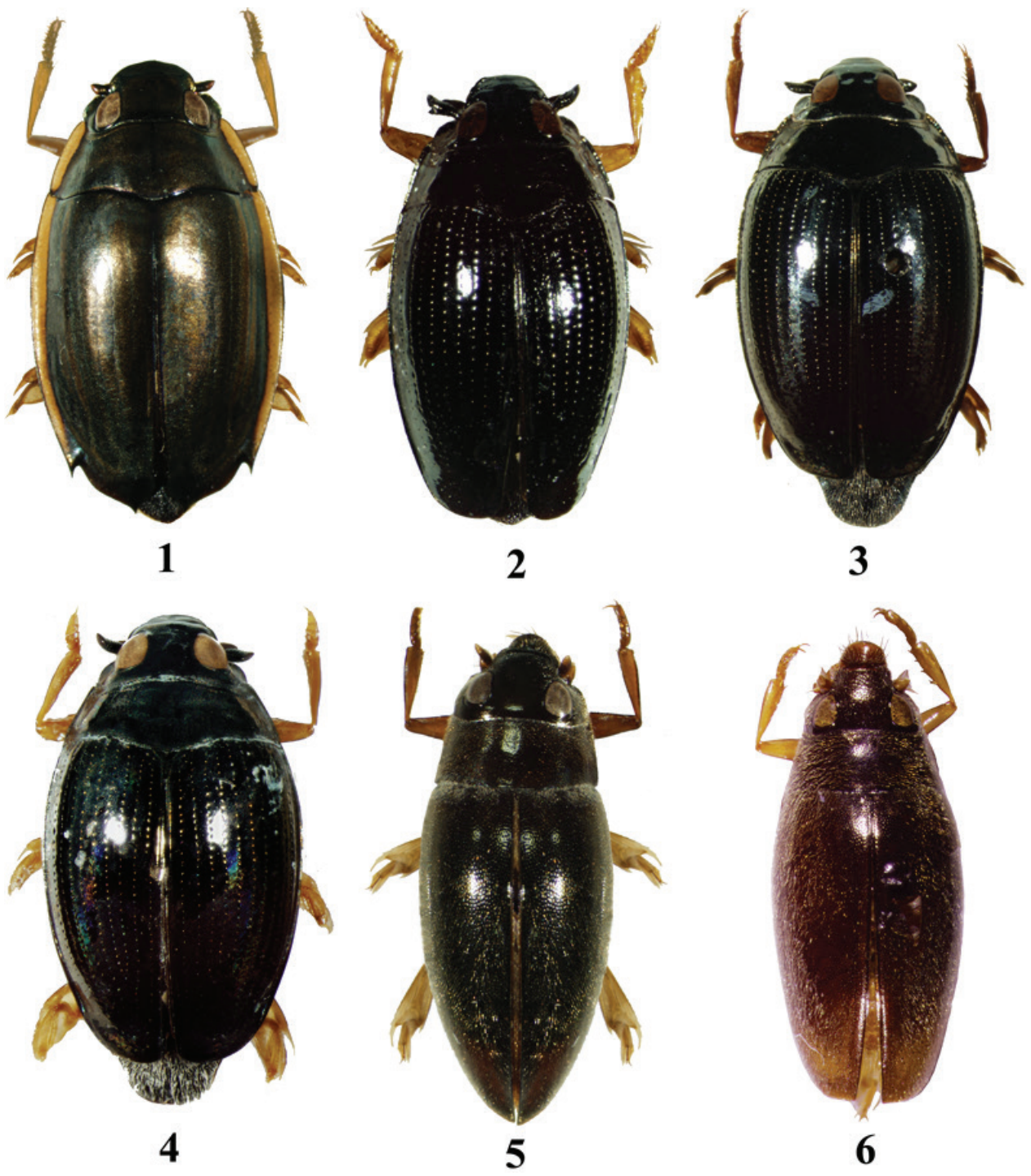

Figures I-6. Habitus. I Dineutus orientalis, $9.0 \mathrm{~mm} 2$ Gyrinus gestroi, $4.8 \mathrm{~mm} 3$ G. japonicus, $7.0 \mathrm{~mm}$ 4 G. pullatus, $6.0 \mathrm{~mm} 5$ Orectochilus punctipennis, $6.5 \mathrm{~mm} 6$ O. villosus, $5.7 \mathrm{~mm}$.

Specimens examined. NORTH KOREA: Gangwon Prov.: 1 $\lesssim 1 \uparrow$, Uonsan-city, Anby-

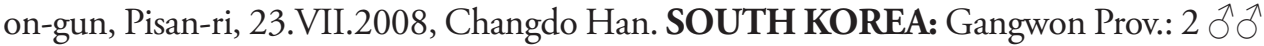
1 ㅇ, Cheorwon-gun, Dongsong-eub, Odeok-ri, Hakji-reservoir, 15.IX.1990; Gyeongbuk Prov.: 3 ô 3 우, Euiseong-gun, Geumseo-myeon, Sujeong-ri, 27.VII.2010, SW Jung,

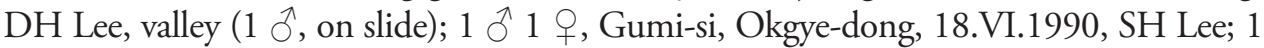
†, Gyeongju-si, Geoncheon-eub, Sinpyeong-ri, 28.V.1993, SH Lee; 1 गे, Gyeongju-si, Wolseong-dong, 5.VI.1987, HM Lee; Gyeonggi Prov.: 1 수 2 우, Hwaseong-si, Songsanmyeon, Dokji-ri, N37²15'25.08", E12640'49.68", 5 m, 4.VII.2013, DH Lee, SG Lee, 
pond near brackish zone; 1 ㅇ, Incheon-si, Ganghwa-gun, Gyodong-myeon, 6.X.2009,

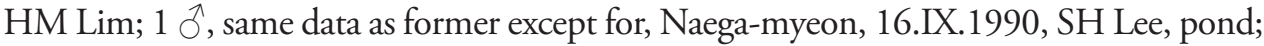
1 ㅇ, Suwon-si, 24.VI.1969; Jeju Prov.: 1 ô, Bukjeju-gun, Jocheon-eub, Seonheul-ri, 11.VI.2005, DH Lee, pond; 1 đ̂, Seoguipo-si, Daejeong-eub, Boseong-ri, 9.VII.1985; Jeonnam Prov.: 1 $\widehat{\jmath}$, Haenam-gun, Hwangsan-myeon, Namri-ri, 13.VI.2010, SH Lee; 1 §ै, Hawsun-gun, Dong-myeon, Jangdong-ri, 3.VIII.2009, SH Lee.

Published Korean records. Dineutus (Spinosodineutes) orientalis: Mazzoldi (1995: 160); Mazzoldi (2003: 26). Dineutus orientalis: Kamiya (1936: 16); Mochizuki and Tsunekawa (1937: 78); Mochizuki and Matsui (1939: 56); Kamiya (1940: 130); Cho (1957: 201); Cho (1963: 46); Cho (1969: 191); Kim and Nam (1982: 25); Lee et al. (1985: 402); Kwon and Suh (1986: 98); Yoon (1988: 617); Lee et al. (1992: 55); Kim et al. (1994: 14); Lee (1995: 15); Hua (2002: 41); Han et al. (2007: 271); Han et al. (2008: 261); Park et al. (2008a: 248); Cho and Park (2010: 95). Dineutus marinatus: Okamoto (1924: 167); Takizawa (1931: 15); Yoshino (1935: 16); Kusanagi (1936: 323).

Diagnosis. Length $9.0-10.0 \mathrm{~mm}$. Dorsal surface mostly dark grey; clypeus, labrum, interorbital area metallic green; lateral parts of pronotum, margin of elytra yellow. Maxillary palpi shorter than labial palpi, palpomere 4 as long as 1-3 combined. Anterior margin of ligula slightly bisinuate; labial palpomere 3 as long as 1-2 combined. Gula with few setae on lateral margin. Postero-lateral and apical parts of elytron sharply pointed (arrows in Fig. 9). Antero-medial margin of prosternum (Fig. 10) strongly rounded. Prosternal process nearly parallel-sided (arrow in Fig. 10). Median lobe of aedeagus (Figs 41-43) shorter than paramere; apical part acute; sperm-groove as in Fig. 42. Paramere (Figs 41, 43) slightly curved at anterior fourth; apical margin rounded.

Distribution. Korea, China (Fujian, Guangdong, Guizhou, Guangxi, Hebei, Jiangsu, Liaoning), Japan, Russia (Far East) (Mazzoldi 2003).

Habitat. Most Korean specimens are found in ponds or mountain streams with plentiful vegetation and low water temperature. Some specimens were collected in ponds near brackish water.

\section{Genus Gyrinus Geoffroy, 1762}

Figs $15-22$

Gyrinus Geoffroy, 1762: 194. Type species: Dytiscus natator Linné, 1758.

Diagnosis. Head (Fig. 15) about 3.0 times wider than long, with micro-reticulation. Frons with two rounded depression (arrows in Fig. 15). Antenna with 9 antennomeres, antennomere 2 rugous with sparse punctures, 9 with compact short setae on apico-lateral part. Clypealium with long setae on antero-lateral part. Galea 1-articled. Pronotum with transverse depression on median parts (arrow in Fig. 16), lateral parts rugous, anterior margin slightly sinuate, posterior margin slightly rounded, anterior angle acute, posterior angle acute. Elytron (Fig. 16) with 11 punctato-striae and with- 


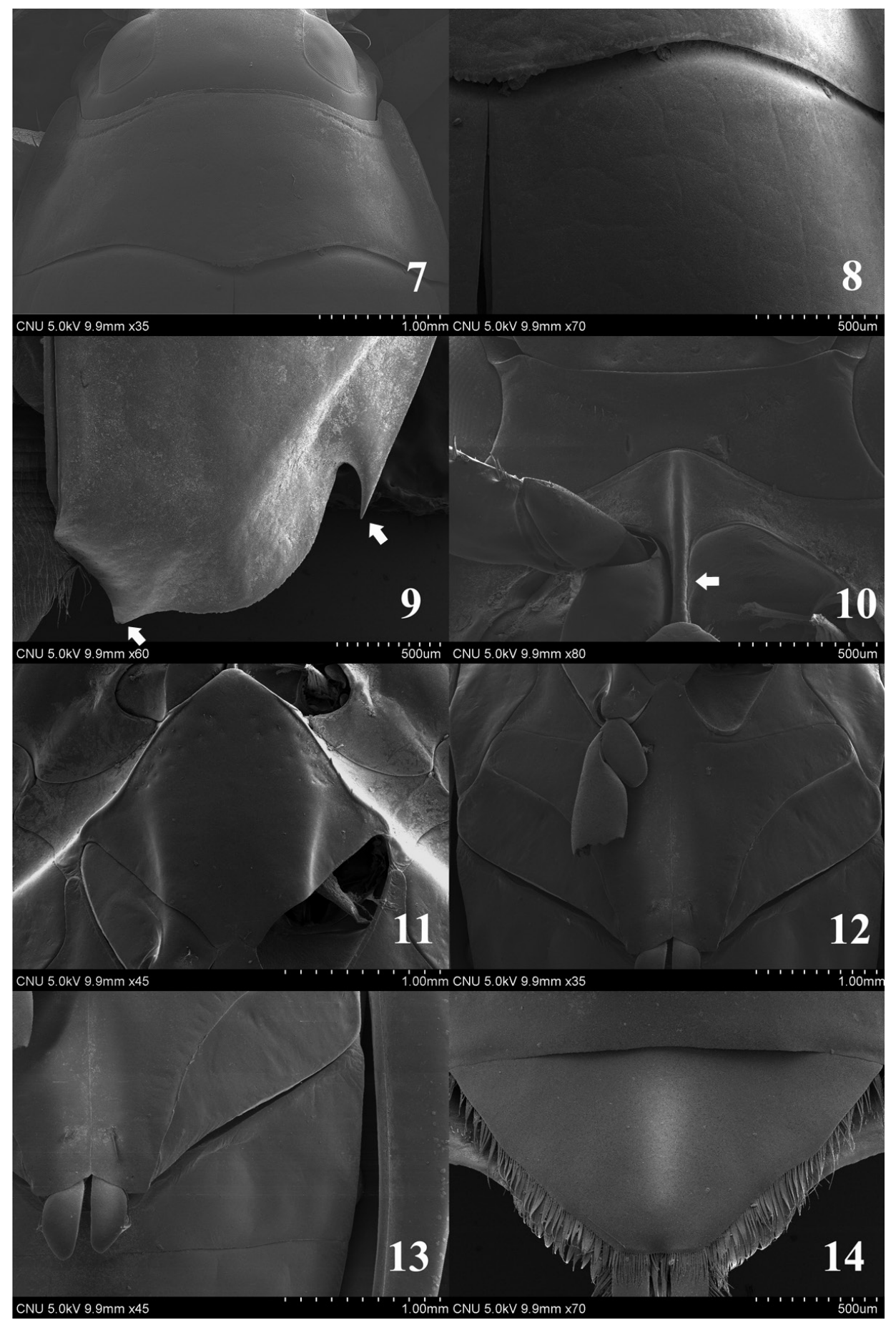

Figures 7-14. Dineutus orientalis. 7 pronotum (dorsal aspect) 8 basal part of elytron (dorsal aspect) 9 apical part of elytron (dorsal aspect) 10 prosternum (ventral aspect) I I mesoventrite (ventral aspect) I 2 metaventrite (ventral aspect) I 3 sternite II (ventral aspect) I 4 sternite VIII (ventral aspect). 
out compact setae, apical margin rounded; subelytral suture present (arrow in Fig. 17); epipleura reached on lateral part of sternite VII, anterior part of epipleura rounded. Prosternum transverse; anterior margin slightly rounded (Fig. 18). Prosternal process (Fig. 18) linear-form, gradually broad at posterior part. Metaventrite (Fig. 19) elongated, diamond-shaped, flattened; anterior part very acute; lateral parts curved upwardly. Tergite VIII (Fig. 20) with compact long setae and posterior margin rounded. Sternites III-VII (Fig. 21) with weak depression on lateral part. Sternite VII (Fig. 21) with long setae on lateral margin. Sternite VIII (Fig. 22) with long setae on postero-lateral parts and posterior margin rounded.

\section{Subgenus Gyrinus Geoffroy, 1762}

Gyrinus Geoffroy, 1762: 194. Type species: Dytiscus natator Linné, 1758.

\section{Key to the species of Korean Gyrinus}

1 Hypomera and epipleura dark brown; apical part of median lobe less than 3.0 times as narrow as basal part ......................................................... 2

- Hypomera and epipleura yellowish brown to brown; apical part of median lobe (Figs 50-52) more than 3.0 times as narrow as basal part.... G. pullatus Median part of mesoventrite (Fig. 23) with deep pit and large groove; median lobe (Figs 44-46) slightly shorter than paramere, apical margin rounded......

G. gestroi

- Median part of mesoventrite (Fig. 26) with shallow pit and vertical plica; median lobe (Figs 47-49) distinctly shorter than paramere, apical margin broadly rounded

G. japonicus

\section{Gyrinus (s. str.) gestroi Régimbart, 1883}

Figs 2, 18, 23-25, 44-46, 58

Gyrinus gestroi Régimbart, 1883: 165.

Specimens examined. SOUTH KOREA: Jeju Prov.: 1 ð̊, Bukjeju-gun, Jocheon-eub, Gyorae-ri, 10.V.1974; 1 ภ 1 q, same data as former except for, 27.VII.2005, DH Lee,

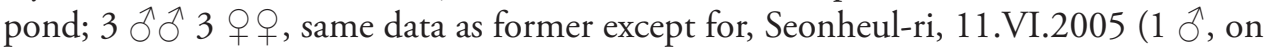
slide); $1 \hat{\jmath}$, same data as former except for, 28.VII.2005, SH Lee, (1 $\hat{\jmath}$, on slide); 2

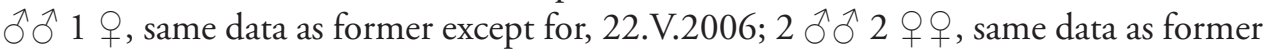
except for, 25.IX.2008; $1 \hat{\jmath}$, same data as former except for, 15.VI.2011 (1 $\hat{\sigma}$, on slide); 2 ઈึ 2 우, Jeju-si, Ara-dong, Gwaneum-temple, 22.VII.1990, SH Lee; 1 , same data as former except for, Jeju National University, 11.VII.1985; 1 ð̊, Seoguiposi, Pyoseon-eub, 10.VII.1985; 1 ㅇ, Seoguipo-si, Seoho-dong, 23.VII.1990, SH Lee, pond; $1 \stackrel{\lambda}{\jmath}$, Seoguipo-si, Seongsan-eub, Ojo-ri, 24.VII.1990, SH Lee. 


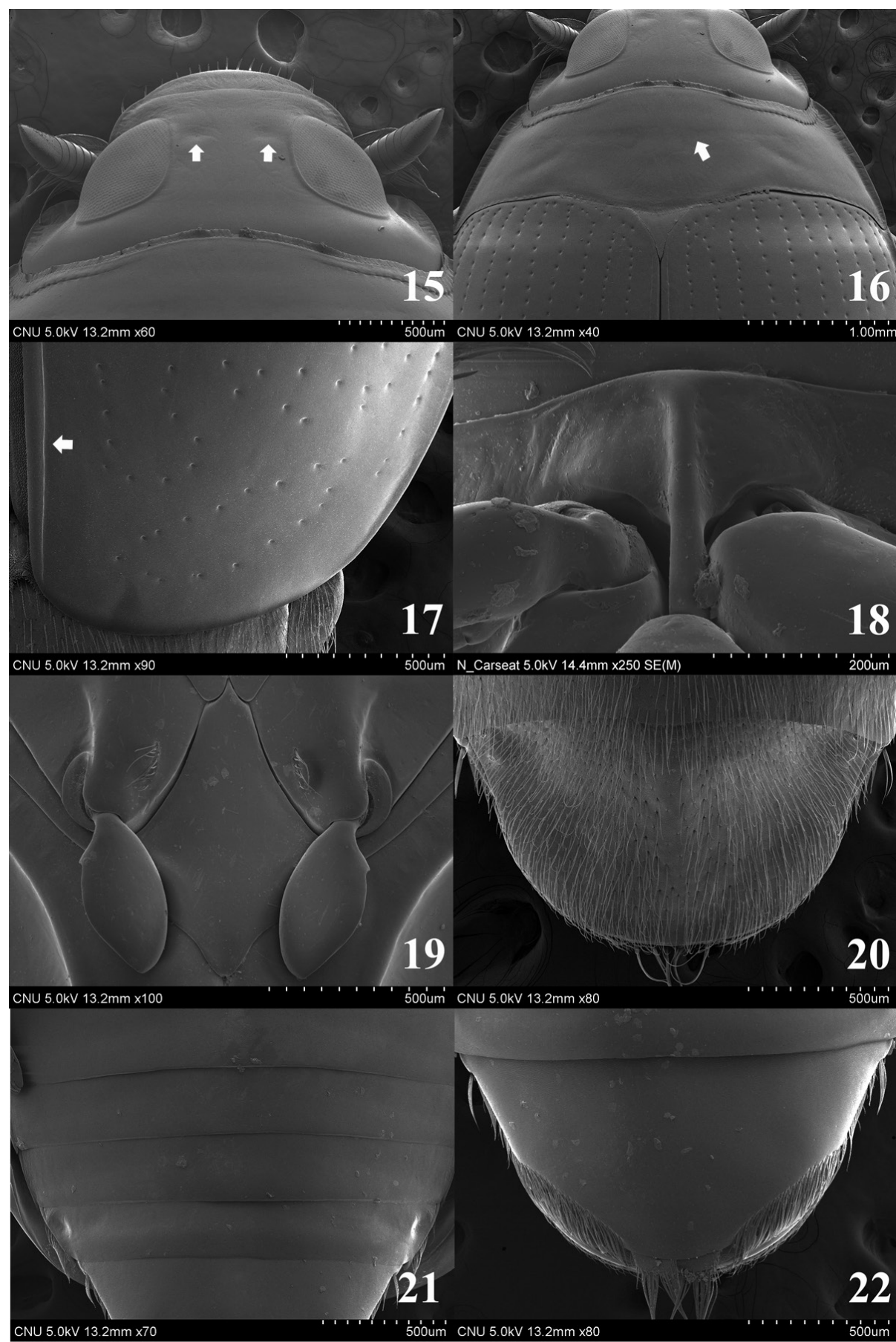

Figures 15-22. 15-17, 19-22 Gyrinus japonicus 18 G. gestori 15 head (dorsal aspect) 16 pronotum (dorsal aspect) $\mathbf{1 7}$ apical part of elytron (dorsal aspect) $\mathbf{1} \mathbf{8}$ prosternum (ventral aspect) $\mathbf{1 9}$ metaventrite (ventral aspect) $\mathbf{2 0}$ tergite VIII (dorsal aspect) $\mathbf{2} \mathbf{I}$ sternites IV-VII (ventral aspect) $\mathbf{2 2}$ sternite VIII (ventral aspect). 
Published Korean records. Gyrinus gestroi: Kwon and Suh (1986: 98); Lee et al. (1992: 54); Kim et al. (1994: 134); Lee (1995: 13); Park et al. (2008: 247); Cho and Park (2010: 95).

Diagnosis. Length 4.5-5.5 mm. Ventral surface mostly black; ventral part of antennomere 2, mouthparts, prosternum, legs, posterior part of sternite VII, sternite VIII yellowish brown to brown; hypomera, epipleura dark brown. Ligula with a row of sparse spines on antero-medial part. Anterior margin of mesoventrite rounded, posterior margin bifid with very deep pit (arrow in Fig. 23), large groove present on postero-medial and antero-lateral parts. Metepisternum with a deep pit (arrow in Fig. 24), oval groove present on anterior part. Sternite II with deep pit (arrow in Fig. 25), transverse groove on anterior part. Median lobe of aedeagus (Figs 44-46) nearly parallel-sided at middle to apex, slightly shorter than paramere; apical margin rounded; sperm-groove as in Fig. 45. Paramere (Figs 44, 46) broader than median lobe; apical margin broadly rounded.

Distribution. Korea, Japan (Mazzoldi 2003).

Habitat. All specimens were collected in ponds with plentiful vegetation in Jejudo Island. They are frequently found together with D. orientalis or G. japonicus.

\section{Gyrinus (s. str.) japonicus Sharp, 1873}

Figs 3, 15-17, 19-22, 26-28, 47-49, 58

Gyrinus japonicus Sharp, 1873: 55.

Gyrinus (Gyrinus) japonicus: Cheo (1934: 210).

Gyrinus japonicus francki Zaitzev, 1953: 355. Synonymized by Mazzoldi (2003: 27).

Type specimens examined. Syntypes: $1 \hat{\jmath} 1$ ( $\mathrm{NHM}$ ), with labels as follows: "Gyrinus japonicus Types D. S. Yokohama. Lewis, Type, Sharp Coll. 1905-313., Japan. G. Lewis.".

Additional material examined. NORTH KOREA: 6 ภ city, Around Pyongyang-Hotel, Near Daedong-River, 15 vii 2008, Changdo Han; 16 ㅊํ 13 우우, Pyongyang-city, Mt. Daesong-San, 24.VI.2009, Changdo Han. SOUTH KOREA: Chungbuk Prov.: 1 ô, Yeongdong-gun, Yongsan-myeon, Sinhang-ri, 9.V.2009, SH Lee, pond; Chungnam Prov.: 1 ô, Daejeon-si, Yusong-gu,

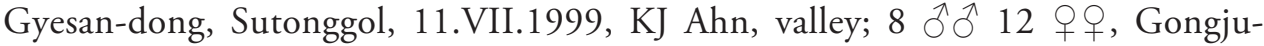
si, Banpo-myeon, Hakbong-ri, Mt. Gyeoryongsan, 25.VII.1992, SH Lee; 2 q , Yesan-gun, Deoksan-myeon, Sacheon-ri, Surak-temple, 26.VII.1995, SH Lee;

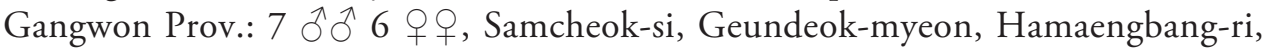
Chodang-reservoir, 7.IX.1990; Gyeongbuk Prov.: 1 \%, Daegu-si, Buk-gu, Baekandong, 4.VI.1985; 13 ㅊํ 12 우우, Gumi-si, Haepyeong-myeon, Songgok-ri, Doritemple, 5.VIII.1994, SH Lee; 1 + , Gumi-si, Okgye-dong, 18.VI.1990, SH Lee; 1

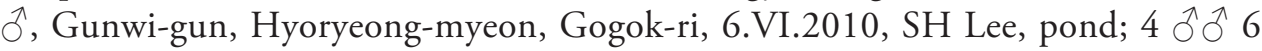
웅, Gyeongju-si, Geoncheon-eub, Sinpyeong-ri, 28.V.1993, SH Lee; 1 으, Gyeo- 


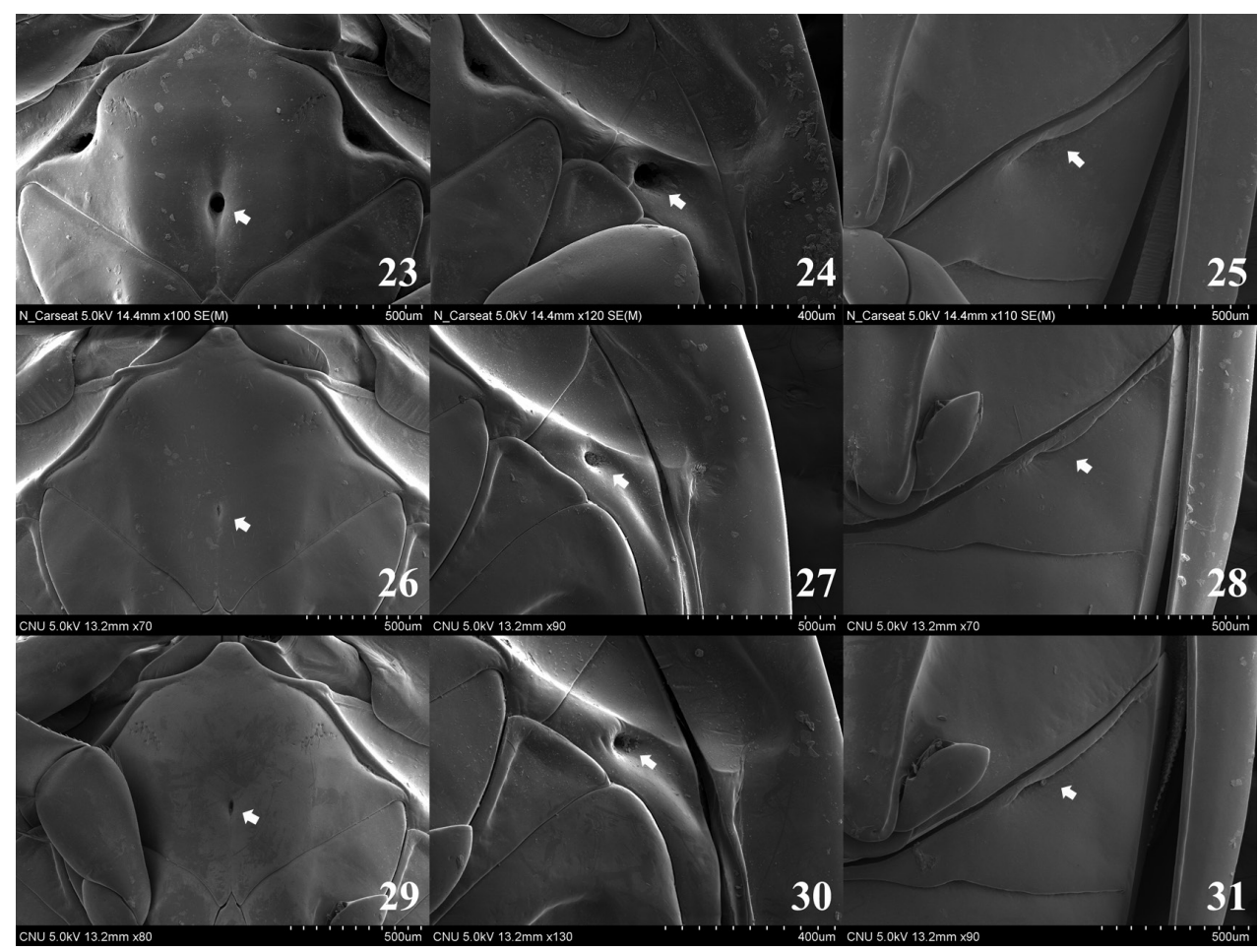

Figures 23-3 I. 23-25 Gyrinus gestroi 26-28 G. japonicus 29-3 I G. pullatus 23 mesoventrite (ventral aspect) $\mathbf{2 4}$ metepisternum (ventral aspect) $\mathbf{2 5}$ sternite II (ventral aspect) $\mathbf{2 6}$ mesoventrite (ventral aspect) $\mathbf{2 7}$ metepisternum (ventral aspect) $\mathbf{2 8}$ sternite II (ventral aspect) $\mathbf{2 9}$ mesoventrite (ventral aspect) $\mathbf{3 0}$ metepisternum (ventral aspect) $\mathbf{3} \mathbf{I}$ sternite II (ventral aspect).

ngju-si, Jinhyeon-dong, Bulguk-temple, 23.VI.1991, SH Lee; 6 ô ô 6 우으, Gyeongju-si, Naenam-myeon, Yongjang-ri, Hawgok-pond, 25.IV.1994, SH Lee, pond; 1 ․ Kimcheon-si, Buhang-myeon, Daeya-ri, 29.VIII.2011, DH Lee, SW Jung, mountain stream; 36 तै 38 우, Pohang-si, Buk-gu, Jukjang-myeon, Duma-ri, 1.X.1989, SH Lee; 1 o 2 우, Pohang-si, Gigye-myeon, Hwabong-ri, 5.IV.1993, SH Lee, pond; $1{ }^{\Uparrow} 1$ 우, Sangju-si, Jungdong-myeon, Osang-ri, 18.VI.1990, SH Lee, pond; 1 ô 3 우, Uljin-gun, Seo-myeon, Wangpi-ri, 23.IV.1994, SH Lee, stream; 1 Oे, Ulsan-si, Ulju-gun, Samnam-myeon, Gacheon-ri 30.VI.2003, YB Cho, MJ

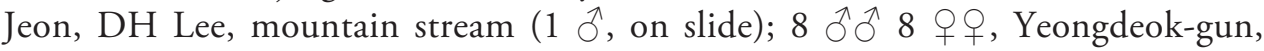
Yeonghae-myeon, Myogok-ri, 6.VI.1994, SH Lee, pond; Gyeonggi Prov.: 1 ôे 1 , Anseong-si, 10.IX.1977, DW Oh; 1 , Pocheon-si, Byeolnae-myeon, Yongam-ri, Mt. Yongamsan [= Sori-bong], 16.VII.1992, SH Lee; 1 +, Seoul-si, Gangbukgu, Ui-dong, Sogui-stream, 23.VI.2007, JG Lee, valley; 1 $\widehat{~}$, Yongin-si, Suji-gu, Sinbong-dong, 19.VII.1988, JH Lee; Gyeongnam Prov.: 1 ô, Busan-si, Seogu, Ulmang-dong, 26.IV.2009, SH Lee; 1 సै, Goseong-gun, Gaecheon-myeon, 
Bukpyeong-ri, 13.VIII.1995, SH Lee; 2 우, Euiryeong-gun, Yongdeok-myeon, Imok-ri, Deokam-pond, 18.V.2009, SH Lee, pond; 2 주 5 웅, Geoje-si, Geo-

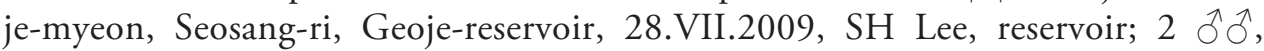
Habcheon-gun, Samga-myeon, Eojeon-ri, 8.V.2009, SH Lee, pond; 1 + , Hamy-

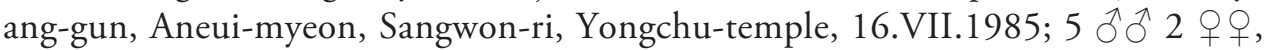
Sacheon-si, Gonmyeong-myeon, Yongsan-ri, Dasol-temple, 14.VIII.1995, SH Lee; 1 \ิ 2 우, Sacheon-si, Sanan-myeon, 13.VIII.1995, SH Lee; $1 \hat{\jmath}$, Sancheonggun, Sancheong-eub, Jeonggok-ri, Jipum-church, 8.V.2009, SH Lee; Jeju Prov.:

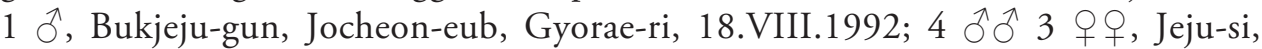
Ara-dong, Gwaneum-temple, 22.VII.1990, SH Lee (1 $\hat{\jmath}$, on slide); $1 \hat{\jmath}$, Jeju-si, Hangyeong-myeon, Yongsu-ri, 17.VII.1992, SH Lee; Jeonbuk Prov.: 20 ठิ 27 우우, Jeongeub-si, Naejang-dong, Mt. Naejangsan, 4.VIII.1990, SH Lee; 2 ठิત 2 우우, Namwon-si, Sandong-myeon, Daesang-ri, Guijeong-temple, 28.VII.2008, DH Lee, pond; 6 ô 6 우오, Namwon-si, Sannnae-myeon, Ibseok-ri, Silsang-temple, 12.VI.2008, DH Lee, pond (1 $\delta^{\lambda}$, on slide); 1 , Namwon-si, Unbong-eub,

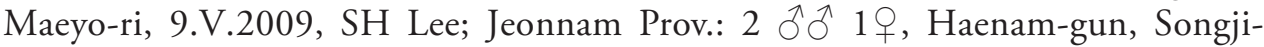
myeon, Geumgang-ri, 27.VII.2010, SH Lee, pond; 1 ते, Gurye-gun, Gurye-eub,

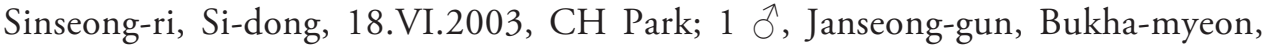
Sinseong-ri, Mt. Naejangsan, 2.IV.2010, JC Jeong (1 $\widehat{~}$, on slide).

Published Korean records. Gyrinus japonicus: Kolbe (1886: 179); Takizawa (1931: 18); Kamiya (1936: 20); Mochizuki and Tsunekawa (1937: 78) Mochizuki and Matsui (1939: 55); Ishii (1940: 43); Cho (1957: 201); Cho (1969: 191); Kim and Nam (1982: 25); Lee et al. (1985: 402); Yoon (1988: 616); Kim and Lee (1991: 65); Lee et al. (1992; 52); Lee (1994: 15); Lee (1995: 13); Mazzoldi (1995: 158); Nilsson et al. (2001: 29); Hua (2002: 41); Mazzoldi (2003: 27). Gyrinus japonicus franki (synonym): Kwon and Suh (1986: 98); Kim et al. (1994: 134); Kim (1995: 132); Han et al. (2007: 271); Han et al. (2008: 259); Cho and Park (2010: 95).

Diagnosis. Length $6.5-8.5 \mathrm{~mm}$. Ventral surface mostly black; mouthparts, prosternum, legs, sternite VIII brown to reddish brown; hypomera, epipleura dark brown. Ligula with a row of compact spines on antero-medial part. Anterior margin of mesoventrite acute and posterior margin bifid; shallow pit (arrow in Fig. 26) and vertical plica present on postero-medial part; shallow plica present on antero-lateral parts. Metepisternum with a pit (arrow in Fig. 27) and shallow groove on anterior part. Sternite II with small pit and transverse plica on anterior part (arrow in Fig. 28). Median lobe of aedeagus (Figs 47-49) parallel-sided at middle to apex, shorter than paramere, narrowest at middle; apical margin nearly straight; sperm-groove as in Fig. 48. Paramere as in Figs 47 and 49.

Distribution. Korea, China (Northeast Territory), Japan, Russia (Far East) (Mazzoldi 2003).

Habitat. Specimens were collected in ponds with plentiful vegetation and low water temperature. In summer, we often found that a large number of individuals gathered whirling on surface of water. 


\section{Gyrinus (s. str.) pullatus Zaitzev, 1908}

Figs 4, 29-31, 44, 46

Gyrinus pullatus Zaitzev, 1908: 244.

Specimens examined. SOUTH KOREA: Gyeongbuk Prov.: 1 d, Gyeongju-si, Jinhyeon-dong, Bulguk-temple, 23.VI.1991, SH Lee; 1 ô, Gyeongju-si, Naenam-myeon, Hwagok-ri, 25.IV.1993, SH Lee, pond; 1 ઈ 1 ㅇ, Pohang-si, Nam-gu, Yeongil-eub, 22.VI.1992, SH Lee; 1 , Uljin-gun, Wonnam-myeon, Maehwa-ri, Maehwa-stream, 10.VI.1995, SH Lee; 1 +, Yeongdeok-gun, Changsu-myeon, Changsu-ri, 5.VI.1994, SH Lee, pond; $2 \widehat{ } \sigma^{\Uparrow}$, Yeongdeok-gun, Yeonghae-myeon, 17.VI.1985, SH Lee; 15 ô d̂ $_{15}$ 우우, Yeongdeok-gun, Yeonghae-myeon, Myogok-ri, 6.VI.1994, SH Lee, pond ( $1 \hat{0}$, on slide).

Published Korean records. Gyrinus pullatus: Holmen (1987: 49); Mazzoldi (1995: 159); Nilsson et al. (2001: 31); Hua (2002: 41); Mazzoldi (2003: 28); Gyrinus curtus (misidentification): Mochizuki and Tsunekawa (1937: 78); Mochizuki and Matsui (1939: 56); Kim and Nam (1982: 25); Kwon and Suh (1986: 98); Kim et al. (1994: 134); Lee (1994: 15); Cho and Park (2010: 95).

Diagnosis. Length $6.0-7.0 \mathrm{~mm}$. Ventral surface mostly reddish brown; ventral part of antennomere 2, mouthparts, prosternum, legs, posterior part of sternite VII, sternite VIII yellowish brown to brown; hypomera, epipleura yellowish brown. Ligula with a row of sparse spines on antero-medial part. Anterior margin of mesoventrite rounded and posterior margin bifid; deep pit (arrow in Fig. 29) and vertical groove present on postero-medial part; deep groove present on antero-lateral parts. Metepisternum with a deep pit (arrow in Fig. 30) on anterior part. Sternite II with pit, transverse and thick plica on anterior part (arrow in Fig. 31). Median lobe of aedeagus (Figs 50-52) narrowed apically, shorter than paramere; narrowest at anterior fifth; apical margin nearly straight; sperm-groove as in Fig. 51. Paramere as in Figs 50 and 52.

Distribution. Asia: Korea, China (Liaoning, Jilin), Russia (East Siberia, Far East), Europe: Estonia, Finland, Russia (North European Territory), Sweden (Mazzoldi 2003).

Remarks. Gyrinus curtus was first recorded in Korea by Mochizuki and Tsunekawa (1937). After that, many entomologists [Mochizuki and Matsui (1939: 56); Kim and Nam (1982: 25); Kwon and Suh (1986: 98); Kim et al. (1994: 134); Lee (1994: 15); Cho and Park (2010: 95)] reported this species in Korea, only in the local fauna without any taxonomic comments. After examining specimens ( $2 \hat{\jmath} \bar{\gamma}$, Yeongdeokgun, Yeonghae-myeon, 17.VI.1985, SH Lee) previously studied by Kwon and Suh (1986), Kim et al. (1994), Lee (1994), and Cho and Park (2010), we found that they had been incorrectly identified and actually represent $G$. pullatus. This species can be distinguished from G. curtus by the hypomera being yellowish brown to brown and the apical margin of the median lobe being nearly straight. 


\section{Genus Orectochilus Dejean, 1833}

Figs 32-40

Orectochilus Dejean, 1833: 59. Type species: Gyrinus villosus Müller, 1776.

Diagnosis. Body long oval, with micro-reticulation, compact setae present on most dorsal part. Labrum semicircular, slightly wider than long, long setae present on anterior margin. Antenna with 9 antennomeres. Pronotum without transverse depression. Scutellum (Fig. 32) transverse and visible when elytra closed. Elytron (Figs 33, 34) with compact setae. Prosternum (Fig. 35) with few setae on anterior part. Prosternal process (arrow in Fig. 35) sagittiform, widest anterior three forth (Fig. 35). Mesoventrite (Fig. 36) with setae on anterior margin, vertical plica on postero-medial part; anterior part acute. Metaventrite cruciform, vertical process on median part (arrow in Fig. 37). Metepisternum (Fig. 36) without pit and groove. Metatibia with two spines of equal length. Sternite II (Fig. 38) without pit, transverse groove present on anterior part. Sternites IV-VI (Fig. 39) with short setae on median parts. Sternites VII-VIII (Figs 39, 40) with compact long setae on medial parts. Sternite VII (Fig. 39) with long setae on posterior margin. Sternite VIII (Fig. 40) longer than wide, long setae present on medial and lateral parts; apex bifid.

\section{Key to the species of Korean Orectochilus}

$1 \quad$ Body less than $7.0 \mathrm{~mm}$; apical part of elytron not protruded; median lobe of aedeagus shorter than paramere ............................................................ 2

- $\quad$ Body more than $7.0 \mathrm{~mm}$; apical part of elytron protruded; median lobe of aedeagus longer than paramere ................................................. regimbarti

2 Body black; apical part of elytra acute in dorsal view; paramere curved at middle, apical margin of gonocoxa (Fig. 56) rounded .............. O. punctipennis

- Body brown; apical part of elytra broadly round in dorsal view; paramere nearly straight, apical margin of gonocoxa (Fig. 57) broadly rounded

O. villosus

\section{Orectochilus punctipennis Sharp, 1884}

Figs 5, 56, 59

Orectochilus punctipennis Sharp, 1884: 449.

Type material examined. Syntype: 1 o (NHM), with labels as follows: "Orectochilus punctipennis. Types D. S. Yokio. Japan. Lewis, Type, Japan. G. Lewis., Sharp Coll. 1905-313."

Additional material examined. SOUTH KOREA: Gangwon Prov.: 1 , Gangneung-si, Okgyeo-myeon, Jusu-ri, 17.VIII.2011, SW Jung. 


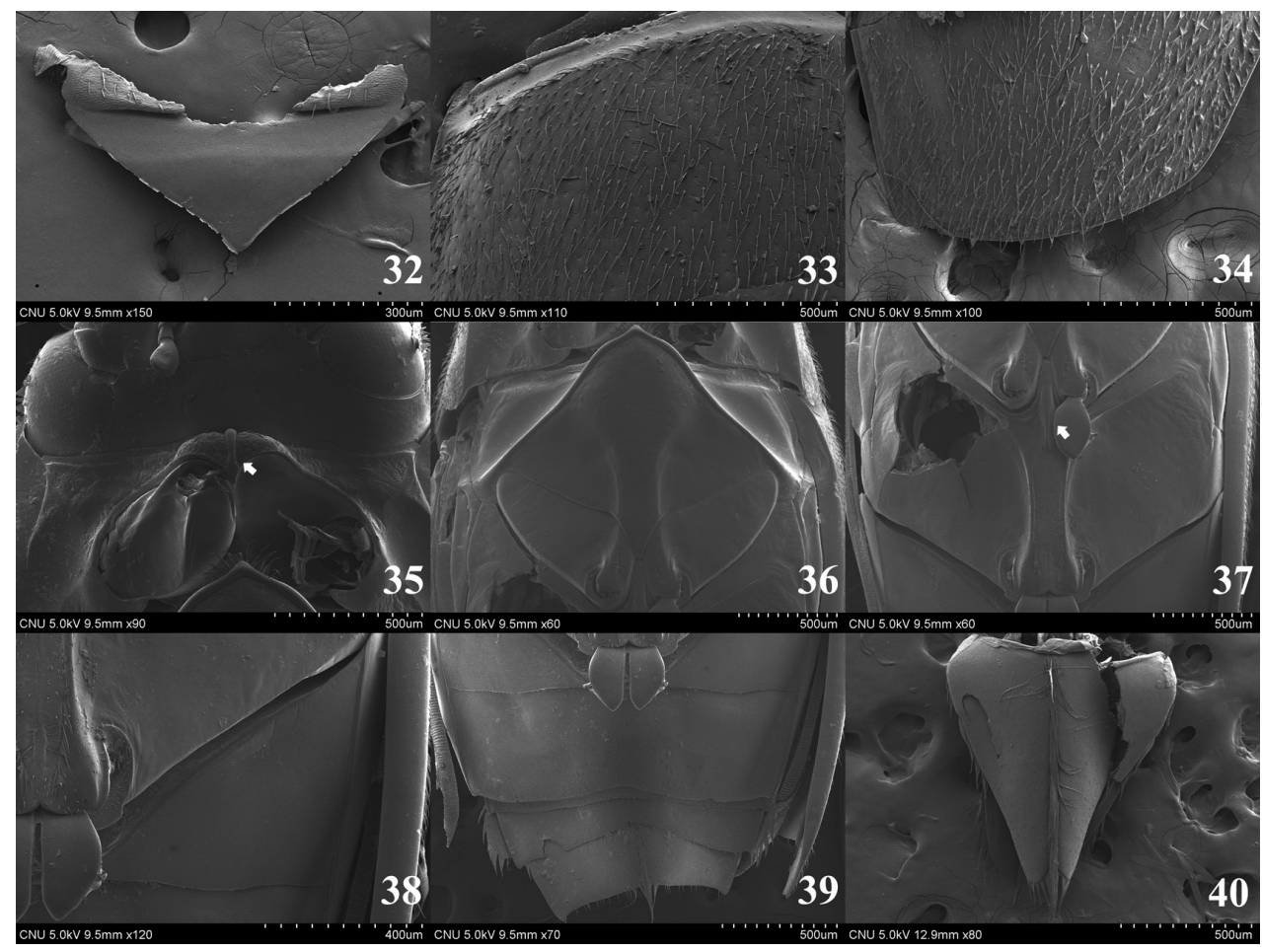

Figures 32-40. Orectochilus villosus. 32 scutellum (dorsal aspect) 33 basal part of elytron (dorsal aspect) $\mathbf{3 4}$ apical part of elytron (dorsal aspect) $\mathbf{3 5}$ prosternum (ventral aspect) $\mathbf{3 6}$ mesoventrite (ventral aspect) $\mathbf{3 7}$ metaventrite (ventral aspect) $\mathbf{3 8}$ sternite II (ventral aspect) $\mathbf{3 9}$ sternites III-VII (ventral aspect) $\mathbf{4 0}$ sternite VIII (ventral aspect).

Diagnosis. Length $6.5 \mathrm{~mm}$. Dorsal and ventral surface mostly black; antenna, mouthparts, hypomera, epipleura, front leg brown; middle and hind legs, sternites V-VII yellowish brown. Head without pubescence on vertex and postero-lateral part. Pronotum widest at posterior margin; anterior angle rectangular; posterior angle rounded. Elytra (Fig. 5) widessst at middle; posterior margin acute in dorsal view. Protarsal claw as long as protarsomere 1. Posterior part of mesoventrite bifid. Median lobe of aedeagus shorter than paramere. Apical margin of gonocoxa (Fig. 56) rounded.

Distribution. Korea, Japan, Russia (Far East) (Mazzoldi 2003).

Remarks. Orectochilus punctipennis is recorded for first time in Korea. This species can be distinguished from $O$. regimbarti by the small size (less than $7.0 \mathrm{~mm}$ ) and median lobe of aedeagus shorter than paramere. It also differs from $O$. villosus by the black body, posterior margin of the elytron acute, paramere curved at middle and apical margin of gonocoxa (Fig. 56) rounded.

Habitat. A single female specimen was collected near the margin of a stream with plentiful vegetation and slow flow velocity. 


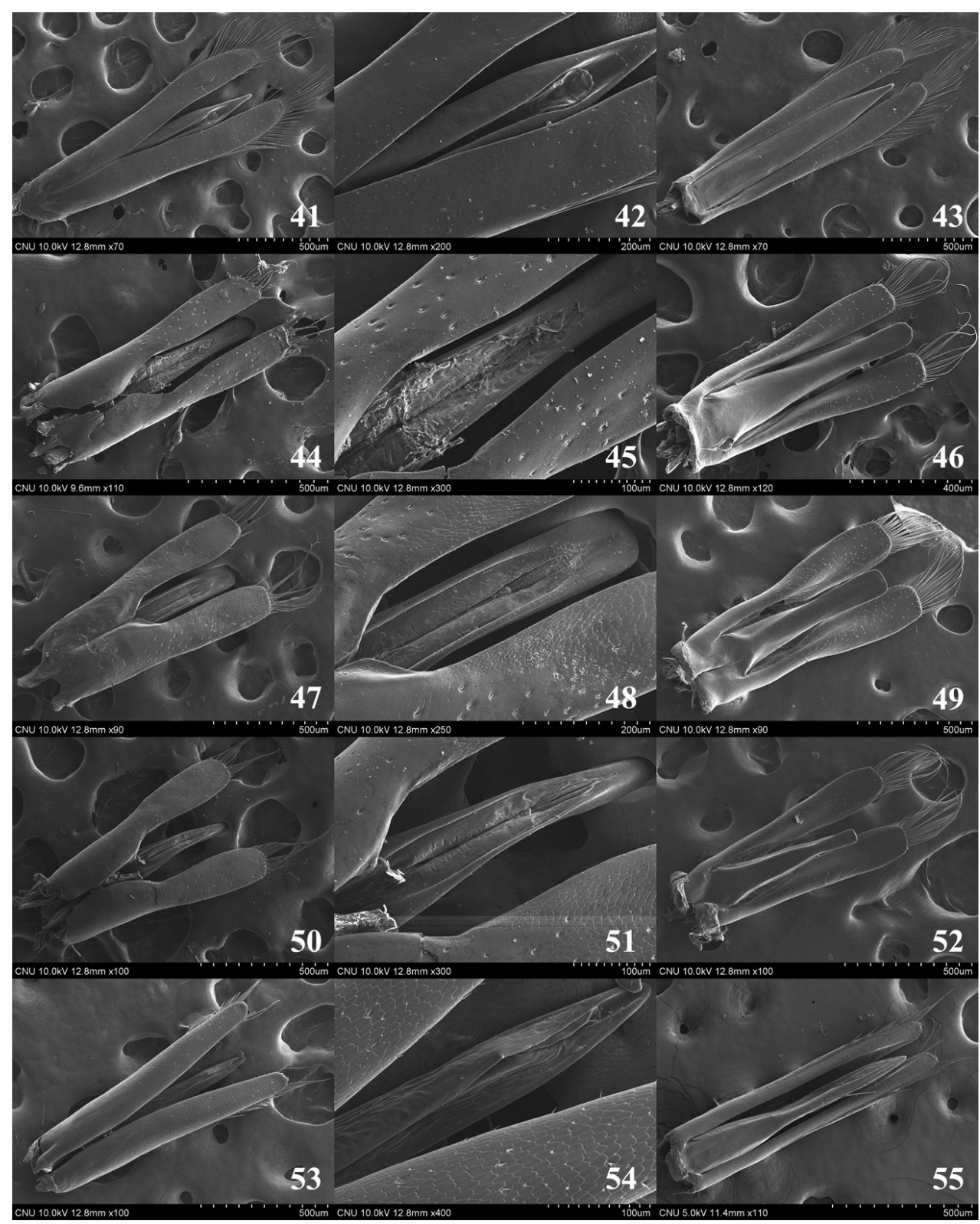

Figures 4I-55. Aedeagus 4I-43 Dineutus orientalis 44-46 Gyrinus gestroi 47-49 G. japonicus 50-52 G. pullatus 53-55 Orectochilus villosus $\mathbf{4 I}$ dorsal aspect $\mathbf{4 2}$ median lobe (dorsal aspect) 43 ventral aspect $\mathbf{4 4}$ dorsal aspect $\mathbf{4 5}$ median lobe (dorsal aspect) $\mathbf{4 6}$ ventral aspect $\mathbf{4 7}$ dorsal aspect $\mathbf{4 8}$ median lobe (dorsal aspect) $\mathbf{4 9}$ ventral aspect $\mathbf{5 0}$ dorsal aspect $\mathbf{5 I}$ median lobe (dorsal aspect) $\mathbf{5 2}$ ventral aspect $\mathbf{5 3}$ dorsal aspect $\mathbf{5 4}$ median lobe (dorsal aspect) $\mathbf{5 5}$ ventral aspect. 

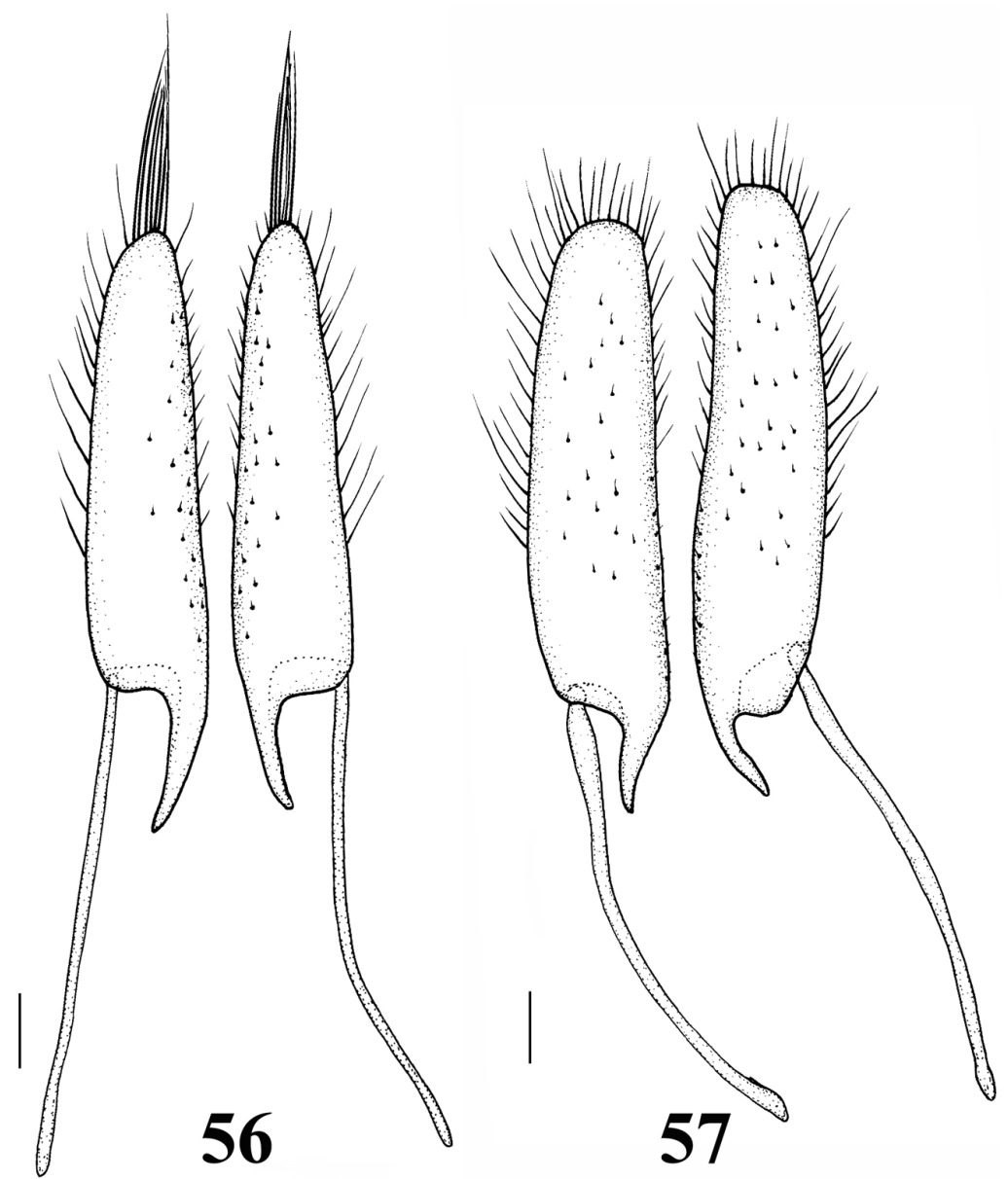

Figures 56-57. Gonocoxa. 56 Orectochilus punctipennis (dorsal aspect) 57 O. villosus (dorsal aspect). Scales $=0.1 \mathrm{~mm}$.

\section{Orectochilus regimbarti Sharp, 1884}

Orectochilus regimbarti Sharp, 1884: 448.

Published Korean records. Orectochilus regimbarti: Kwon and Suh (1986: 99); Kim et al. (1994: 134); Cho and Park (2010: 95).

Distribution. Korea, Japan, Russia (Far East) (Mazzoldi 2003).

Remarks. This species has been recorded in Korea by Kwon and Suh (1986), Kim et al. (1994) and Cho and Park (2010), only in their checklists without any taxonomic comments and specimens. We could not find any Korean specimens and the occurrence of this species in Korea is suspicious. However, its occurrence in Korea is possible because it is known from Japan and Russia (Far East). 


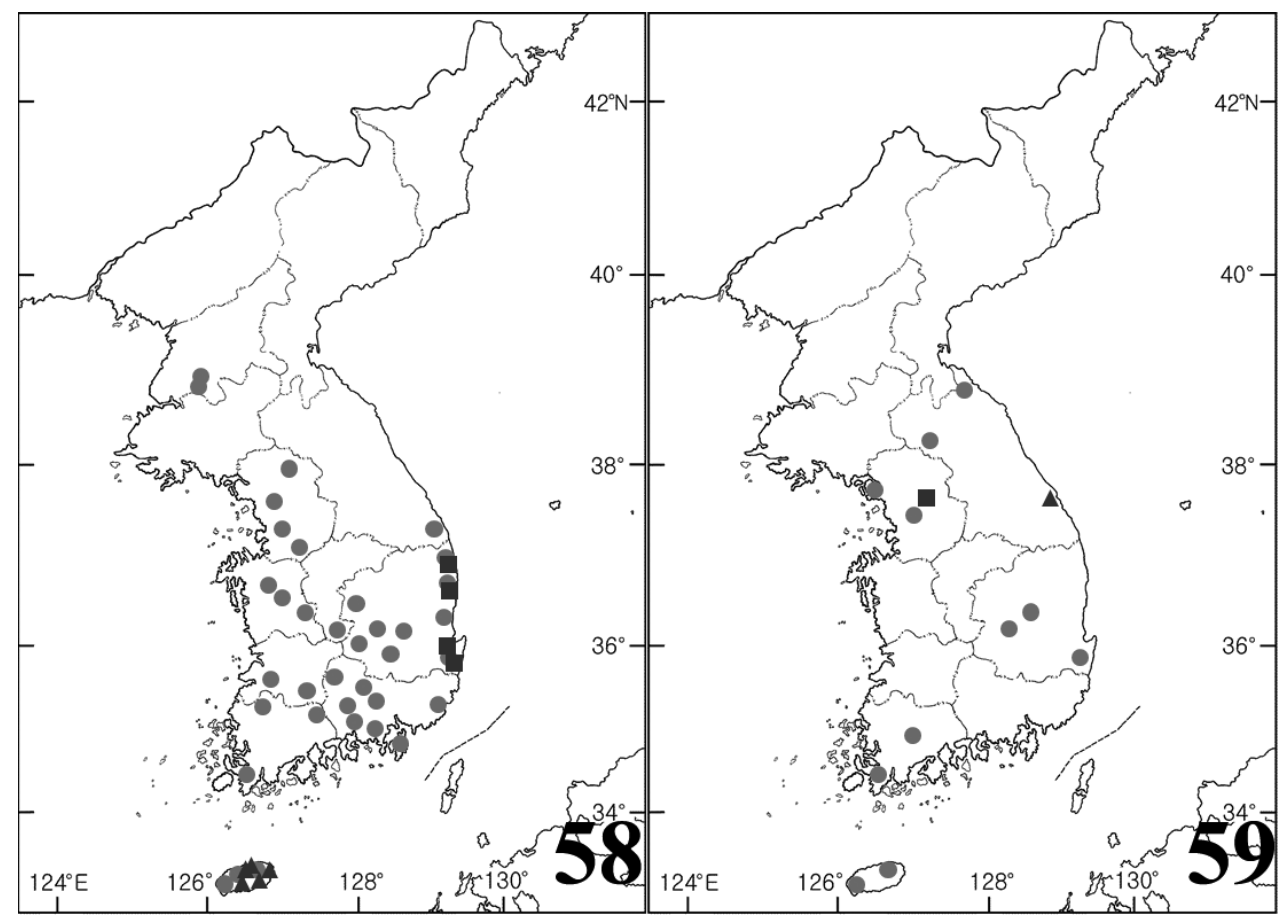

Figures 58-59. Distribution maps. 58 Gyrinus japonicus (circle), G. gestroi (triangle), G. pullatus (square) 59 Dineutus orientalis (circle), Orectochilus punctipennis (triangle), O. villosus (square).

\section{Orectochilus villosus (Müller, 1776)}

Figs 6, 32-40, 53-55, 57, 59

Gyrinus villosus Müller, 1776: 68.

Gyrinus modeeri Marsham, 1802: 100. Synonymized by Illiger (1807: 299).

Orectochilus villosus: Dejean 1883: 59.

Specimens examined. SOUTH KOREA: Gyeonggi Prov.: 2 ๙ึ 3 \% $q$, Namyangjusi, Wabu-eub, Paldang-ri, Paldang-lake, 10.VI.1962, JS Lee (1 ô 1 + , on slide).

Published Korean records. Orectochilus villosus: Yoon (1988: 615); Kim et al. (1994: 134); Cho and Park (2010: 96).

Diagnosis. Length 5.5-6.5 mm. Dorsal surface dark brown; ventral surface mostly brown; ventral part of antennomere 2, mouthparts, hypomera, epipleura; legs yellowish brown. Head without pubescence on postero-lateral margins. Posterior angle of pronotum rectangular. Elytra (Fig. 34) widest at middle, posterior margin broadly rounded. Posterior part of mesoventrite (Fig. 36) slightly acute. Median lobe of aedeagus (Figs 53-55) slender, shorter than paramere; apical part acute; sperm-groove as in Fig. 54. Paramere (Figs 53,55) nearly straight, long setae present on lateral and apical parts; apical part rounded. Apical margin of gonocoxa (Fig. 57) broadly rounded. 
Distribution. Europe, Asia; Korea, China (Liaoning), Cyprus, Iran, Iraq, Israel, Japan, Kazakhstan, Russia (East Siberia, Far East, West Siberia), Syria, Turkey, Uzbekistan (Mazzoldi 2003).

\section{Acknowledgments}

We thank C. Taylor (NHM, London), S. H. Lee (Acheon Elementary School, Gimcheon), S. W. Jung (Korea University, Seoul), G. T. Gustafson (University of New Mexico, New Mexico) and H. Yoshitomi (EUMJ, Matsuyama) for providing many specimens including type series and valuable literature. We are also grateful to $\mathrm{M}$. Fikáček (National Museum, Prague) for his supports and encouragements. Comments by two anonymous reviewers significantly improved the manuscript. Again, we are in debt to H. Frank (University of Florida, Gainesville) for his proofreading a manuscript. Financial support was provided by the National Institute of Biological Resources (NIBR, Incheon) of Ministry of Environment, KOREA (1834-302) and the research on two gyrinid species ( $G$. pullatus and $O$. punctipennis) newly discovered in South Korea was supported by a grant from the National Institute of Biological Resources (NIBR), funded by the Ministry of Environment (MOE) of the Republic of Korea (NIBR 201501203).

\section{References}

Ahlwarth K (1910) Pars 21. Gyrinidae. Coleopterorum Catalogus. Tome IV, Pars. 21, Berlin, $42 \mathrm{pp}$.

Aubé C (1838) Species général des Hydrochanthares et Gyriniens, pour faire suite au species général des Coléoptères de la collection de M. Le Comte Dejean. Méquignon Père et fils, Libraires-éditeurs, Paris, 803 pp.

Brinck P (1955) A revision of the Gyrinidae (Coleoptera) of the Ethiopian region. I. Acta Universitatis Lundensis, Nova Series 51(16): 1-144.

Cheo MT (1934) The Gyrinidae of China. Peking Natural History Bulletin 8: 205-237 + 202 pls. Cho PS (1957) A Systematic Catalogue of Korea Coleoptera. Humanities and Sciences, Korea University 2: 173-338. [In Korean]

Cho PS (1963) Insects of Quelpart Island (Cheju-do). Humanities and Sciences, Korea University 6: 159-242. [In Korean]

Cho PS (1969) Illustrated Encyclopedia of Fauna and Flora of Korea, Vol. 10, Insecta (II). Samhwa Publishing Company, Seoul, 969 pp. [In Korean]

Cho YB, Park SW (2010) Coleoptera. In: Paek MK (Eds) Checklist of Korean Insects. Nature and Ecology, Seoul, 83-157.

Dejean PFMA (1833) Catalogue des Coléoptères de la collection de M. Le comte Dejean. Chez Mélquignon-Marvis Père et fils, Paris, 433 pp. 
Fabricius JC (1781) Species Insectorum enhibentes eorum differenties specificas, synonyma, auctorum, loca Natalia, meramorphosim adiectis observationibus, descriptionibus. Tomus I. C.E. Bohn, Hamburg et Kilonii, vii + 552 pp.

Fairmaire L (1878) [new species]. In: Deyrolle H, Fairmaire L. Descriptions de coléoptères recueillis par M. l'abbé David dans la Chine dentrale. Annales de la Société Entomologique de France 5: 87-140.

Han MS, Na YE, Bang HS, Kim MH, Kim KM, Roh KA, Lee JT (2007) The Fauna of Aquatic Invertebrates in Paddy Field. Korean Journal of Environmental Agriculture 26(3): 267-273. doi: 10.5338/KJEA.2007.26.3.267

Han MS, Na YE, Bang HS, Kim MH, Kang KK, Hong HK, Lee JT, Ko BG (2008) An Illustrated book of Aquatic Invertebrates in Paddy Field (a Revised Edition). National Institute of Agricultural Science and Technology, RDA, Suwon, 416 pp. [In Korean]

Hatch MH (1925) The phylogeny and phylogenetic tendencies of Gyrinidae. Papers of the Michigan Academy of Sciences, Arts and Letters 5: 429-467.

Holmen M (1987) The aquatic Adephaga (Coleoptera) of Fennoscandia and Denmark. I. Gyrinidae, Haliplidae, Hygrobiidae and Noteridae. Fauna Entomologica Scandinavica 20: 1-157.

Hua LZ (2002) List of Chinese Insects Vol. II. Zhongshan University Press, Guangzhou, 612 pp. Illiger JKW (1807) Nachlese zu den Bemerkungen und Zusätzen zu Fabricii Systema Eleutheratorum. Magazin für Insecktenkunde 6: 296-317.

Ishii S (1940) List of Coleoptera kept in Science group of Keityu. Science Report, Keityu 5: 38-60. Jung SW, Lee DH, Ham SA, Huh JM, Hwang JM, Bae YJ (2011) Revised Checklist of the Korean Aquatic Insects. Entomological Research Bulletin 27: 37- 51.

Kamiya K (1936) Family Gyrinidae Family Haliplidae. Fauna Nipponica 10(4/8): 1-55.

Kamiya K (1940) Aquatic beetles of Manchoukuo. Report of the Limnological Survey of Kwangtung and Manchoukuo, Otzu 1940: 113-139.

Kim CW, Nam SH (1982) Insect fauna of Seoul city area. Science and Technology, Korea University 23: 1-52. [In Korean]

Kim JI (1995) Fauna of Coleoptera and Diptera (Insecta) from Pyonsan Peninsula National Park. The Report of the KACN 34: 129-145. [In Korean]

Kim JI, Lee OJ (1991) Changes in insect fauna due to urbanization of Suwon city. Bulletin of the KACN Series 11: 49-105. [In Korean]

Kim JI, Kwon YJ, Paek JC, Lee SM, An SR, Park HC, Chu HY (1994) Order 23. Coleoptera. In: The Entomological Society of Korea and Korean Society of Applied Entomology (Eds) Check List of Insects from Korea. Kon-Kuk University Press, Seoul, 117-214.

Kolbe HJ (1886) Beiträge zur kenntnis der Coleopteren-Fauna Koreas. Archiv für Naturgeschichte 52: 139-240.

Kusanagi T (1936) A list of Coleoptera from Shikoku. The Entomological World 4 (27): 297-329. Kwon YJ, Suh SJ (1986) Check list of Water Beetles from Korea. Insecta Koreana 6: 91-113. Latreille PA (1810) Considerations generales sur l'ordre naturel des animaux composant les classes des crustaces, des arachnides, et des insectes; avec un tableau methodique de leurs genres, disposes en familles. Chez F. Schoell, Paris, 444 pp.

Lee SH (1994) Water beetles of Hyeong San river. Corentomon 5(1): 14-21. [In Korean] 
Lee SH (1995) Water beetles of Bulyeong valley. Corentomon 6(1): 11-16. [In Korean]

Lee SH, Cho YB, Lee CE (1992) The water beetles of Quelpart Island (Coleoptera). Nature and Life 22(2): 45-60. [In Korean]

Lee YJ, Kim WT, Kim TH (1985) Insect Fauna of Mt. Halla. Report of the Academic Survey of Hallasan (Mountain). National Preserve, 351-455. [In Korean]

Linné C von (1758) Systema naturae per regna tria naturae, secundum classes, ordines, genera, species, cum characteribus, differentiis, synonymis, locis. Editio decimal, reformata. Tomus I. Laurentii Salvii, Stockholm, iv + 824 pp. doi: 10.5962/bhl.title.542

Löbl I, Smetana A (2003) Catalogue of Palaearctic Coleoptera, Volume 1, Achostemata, Myxophaga, Adephaga. Apollo books, Stenstrup, 819 pp.

MacLeay WS (1825) Annulosa Javanica or an attempt to illustrate the natural affinities and analogies of the Insects collected in Java by Thomas Horsfield, M.D. F.L. and G.S. and deposited by him in the museum of the honourable East-India Company. Number I. Kingsbury, Parbury and Allen, London, xii +150 pp.

Marsham T (1802) Entomologia Britannica, sistens insect Britammiae indigena, secundum methodum Linnaeanam disposita. Tomus I. Coleoptera. White, London, xxxi + 548 pp. doi: 10.5962/bhl.title.65388

Mazzoldi P (1995) Gyrinidae: Catalog of Chinese Gyrinidae (Coleoptera). In: Jäch MA, Ji L (Eds) Water Beetles of China, Volume I. Zoologisch-Botanische Gesellschaft in Österreich and Wiener Coleopterologenverein, Wien, 155-172.

Mazzoldi P (2003) Family Gyrinidae. In: Löbl, Smetana (Eds) Catalogue of Palaearctic Coleoptera, Volume 1, Achostemata, Myxophaga, Adephaga. Apollo books, Stenstrup, 26-30.

Miller KB, Bergsten J (2012) Phylogeny and classification of whirligig beetles (Coleoptera: Gyrinidae): relaxed-clock model outperforms parsimony and time-free Baysian analyses. Systematic Entomology (2012) 37: 706-746. doi: 10.1111/j.1365-3113.2012.00640.x

Mochizuki M, Tsunekawa W (1937) A list of Coleoptera from Middle-Korea. Journal of the Chosen Natural History Society 22: 75-93.

Modeer A (1776) Anmärkningar anglende Slägtet Gyrinus. Physiographiska Sälskapets Handlingar 1: 155-162.

Mochizuki M, Matsui S (1939) List of remains of Mr. Matsuo. Science Report, Keityu 4: $51-78$.

Müller OF (1764) Fauna Insectorum Fridrichsdalina. Gleditsch, Hafniae et Lipsiae, xxiv + 96 pp.

Müller OF (1776) Zoologiae Danicae prodromus, seu animalium Daniae et Norvegiae indigeneraum characters, nomina, et synonyma imprimis popularium. Hallageriis, Hafniae, xxxii + 282 pp. doi: 10.5962/bhl.title. 13268

Nilsson AN, Lundmark M, Kholin SK, Minakawa N (2001) A faunistic review of the Gyrinus species of the Far East of Russia (Coleoptera: Gyrinidae). Koleopterologische Rundschau 71: $27-35$.

Ochs G (1926) Die Dineuti. 2. Tribus der Unterfam. Enhydrinae, Fam, Gyrinidae (Col.). Entomologische Zeitschrift (Frankfrut) 40: 61-74, 112-126, 129-140, 190-197.

Ochs G (1929) On some new and interesting species of water beetles of the family Gyrinidae in the United States National Museum. Proceedings of the United States National Museum 75 (3): 1-6. doi: 10.5479/si.00963801.2774 
Ochs G (1930) Gyrinoidea. Catalogue of Indian Insects 19: 1-39.

Ochs G (1942) Über die von Herrn J Klapperich in China 1937-1938 gesammelten Gyriniden (Col.). Mitteilungen der Münchner Entomologischen Gesellschaft 32(1): 201-207.

Okamoto H (1924) The insect fauna of Quelpart Island (Saishiu-to). Bulletin of the Agricultural Experimental Station Chosen 1(2): 47-233.

Park HC, Sim HS, Hwang JH, Kang TH, Lee HA, Lee YB, Km MA, Kim JG, Hong SJ, Seol KY, Kim N, Kim SH, Ahn NH, Oh CK (2008) A Field Guide to Aquatic Insects of Rural Environments in Korea. National Institute of Agricultural Science and Technology, RDA, Suwon, 349 pp. [In Korean]

Sharp D (1873) The water beetles of Japan. The Transactions of the Entomological Society of London 1873: 45-67.

Sharp D (1884) The water beetles of Japan. The Transactions of the Entomological Society of London 1884: 439-464.

Slipinski SA, Leschen RAB, Lawrence JF (2011) Order Coleoptera Linnaeus, 1758. In: Zhang ZQ (Ed.) Animal biodiversity: An outline of higher level classification and survey of taxonomic richness. Zootaxa 3148: 203-208.

Takizawa M (1931) The Gyrinidae of Japan. Insecta Matsumurana 6: 13-21.

Yoon IB (1988) Aquatic Insects, Illustrated Encyclopedia of Fauna and Flora of Korea, Volume 30. State-Compiled Textbook Company Limited, Seoul, 840 pp. [In Korean]

Yoshino T (1935) A list of Coleoptera from Zenranando, Chosen, Part 1. Insect World 39(450): $13-17$.

Zaitzev FA (1908) Übersicht der Paläarktischen Vertreter der Gattung Gyrinus (Linn.) Rég. (Coleoptera, Gyrinidae). Russkoe Entomologischeskoe Obozrenie 7[1907]: 238-244.

Zaitzev FA (1953) Fauna of the U.S.S.R. Coleoptera, Vol. 4. Amphizoidae, Hygrobiidae, Haliplidae, Dytiscidae, Gyrinidae. English Translation (1972). Israel Program for Scientific Translations, Jerusalem, $401 \mathrm{pp}$. 